\title{
Measurement of the Fluctuations in the Number of Muons in Extensive Air Showers with the Pierre Auger Observatory
}

A. Aab, ${ }^{1}$ P. Abreu, ${ }^{2}$ M. Aglietta, ${ }^{3,4}$ J. M. Albury,${ }^{5}$ I. Allekotte, ${ }^{6}$ A. Almela,${ }^{7,8}$ J. Alvarez-Muñiz, ${ }^{9}$ R. Alves Batista, ${ }^{1}$ G. A. Anastasi, ${ }^{10,4}$ L. Anchordoqui, ${ }^{11}$ B. Andrada ${ }^{7}$ S. Andringa, ${ }^{2}$ C. Aramo, ${ }^{12}$ P. R. Araújo Ferreira, ${ }^{13}$ H. Asorey, ${ }^{7}$ P. Assis, ${ }^{2}$ G. Avila, ${ }^{14}$ A. M. Badescu, ${ }^{15}$ A. Bakalova, ${ }^{16}$ A. Balaceanu, ${ }^{17}$ F. Barbato, ${ }^{18,12}$ R. J. Barreira Luz, ${ }^{2}$ K. H. Becker, ${ }^{19}$ J. A. Bellido, ${ }^{5}$ C. Berat, ${ }^{20}$ M. E. Bertaina,${ }^{10,4}$ X. Bertou, ${ }^{6}$ P. L. Biermann, ${ }^{21}$ T. Bister, ${ }^{13}$ J. Biteau, ${ }^{22}$ J. Blazek, ${ }^{16}$ C. Bleve, ${ }^{20}$ M. Boháčová, ${ }^{16}$ D. Boncioli, ${ }^{23,24}$ C. Bonifazi, ${ }^{25}$ L. Bonneau Arbeletche, ${ }^{26}$ N. Borodai, ${ }^{27}$ A. M. Botti, ${ }^{7}$ J. Brack, ${ }^{28}$ T. Bretz,${ }^{13}$ F. L. Briechle, ${ }^{13}$ P. Buchholz, ${ }^{29}$ A. Bueno, ${ }^{30}$ S. Buitink, ${ }^{31}$ M. Buscemi,${ }^{32,33}$ K. S. Caballero-Mora, ${ }^{34}$ L. Caccianiga, ${ }^{35,36}$ A. Cancio, ${ }^{8,7}$ F. Canfora, ${ }^{1,37}$ I. Caracas, ${ }^{19}$ J. M. Carceller, ${ }^{30}$ R. Caruso, ${ }^{32,33}$ A. Castellina, ${ }^{3,4}$ F. Catalani,${ }^{38}$ G. Cataldi, ${ }^{39}$ L. Cazon, ${ }^{2}$ M. Cerda, ${ }^{40}$ J. A. Chinellato, ${ }^{41}$ K. Choi,${ }^{42}$ J. Chudoba,${ }^{16}$ L. Chytka ${ }^{43}$ R. W. Clay, ${ }^{5}$ A. C. Cobos Cerutti, ${ }^{44}$ R. Colalillo, ${ }^{18,12}$ A. Coleman, ${ }^{45}$ M. R. Coluccia, ${ }^{46,39}$ R. Conceição, ${ }^{2}$ A. Condorelli, ${ }^{47,24}$ G. Consolati, ${ }^{36,48}$ F. Contreras, ${ }^{14}$ F. Convenga ${ }^{46,39}$ C. E. Covault,${ }^{49,}$ S. Dasso ${ }^{50,51}$ K. Daumiller, ${ }^{52}$ B. R. Dawson, ${ }^{5}$ J. A. Day, ${ }^{5}$ R. M. de Almeida, ${ }^{53}$ J. de Jesús, ${ }^{7,52}$ S. J. de Jong, ${ }^{1,37}$ G. De Mauro, ${ }^{1,37}$ J. R. T. de Mello Neto, ${ }^{25,54}$ I. De Mitri, ${ }^{47,24}$ J. de Oliveira, ${ }^{53}$ D. de Oliveira Franco, ${ }^{41}$ V. de Souza, ${ }^{55}$ E. De Vito, ${ }^{46,39}$ J. Debatin, ${ }^{56}$ M. del Río, ${ }^{14}$ O. Deligny, ${ }^{57}$ H. Dembinski ${ }^{52}$ N. Dhital, ${ }^{27}$ A. Di Matteo, ${ }^{4}$ C. Dobrigkeit, ${ }^{41}$ J. C. D'Olivo, ${ }^{58}$ R. C. dos Anjos, ${ }^{59}$ M. T. Dova ${ }^{60}$ J. Ebr, ${ }^{16}$ R. Engel, ${ }^{56,52}$ I. Epicoco, ${ }^{46,39}$ M. Erdmann, ${ }^{13}$ C. O. Escobar, ${ }^{61}$ A. Etchegoyen, ${ }^{7,8}$ H. Falcke, ${ }^{1,62,37}$ J. Farmer, ${ }^{63}$ G. Farrar, ${ }^{64}$ A. C. Fauth, ${ }^{41}$ N. Fazzini, ${ }^{65}$ F. Feldbusch, ${ }^{66}$ F. Fenu, ${ }^{10,4}$ B. Fick, ${ }^{67}$ J. M. Figueira, ${ }^{7}$ A. Filipčič, ${ }^{68,69}$ T. Fodran, ${ }^{1}$ M. M. Freire,${ }^{70}$ T. Fujii, ${ }^{63, \$}$ A. Fuster, ${ }^{7,8}$ C. Galea, ${ }^{1}$ C. Galelli, ${ }^{35,36}$ B. García, ${ }^{44}$ A. L. Garcia Vegas, ${ }^{13}$ H. Gemmeke, ${ }^{66}$ F. Gesualdi, ${ }^{7,52}$ A. Gherghel-Lascu, ${ }^{17}$ P. L. Ghia, ${ }^{57}$ U. Giaccari, ${ }^{1}$ M. Giammarchi, ${ }^{36}$ M. Giller, ${ }^{71}$ J. Glombitza, ${ }^{13}$ F. Gobbi,${ }^{40}$ F. Gollan, ${ }^{7}$ G. Golup, ${ }^{6}$ M. Gómez Berisso, ${ }^{6}$ P. F. Gómez Vitale, ${ }^{14}$ J. P. Gongora, ${ }^{14}$ N. González, ${ }^{7}$ I. Goos, ${ }^{6,52}$ D. Góra, ${ }^{27}$ A. Gorgi, ${ }^{3,4}$ M. Gottowik, ${ }^{19}$ T. D. Grubb, ${ }^{5}$ F. Guarino, ${ }^{18,12}$ G. P. Guedes, ${ }^{72}$ E. Guido, ${ }^{4,10}$ S. Hahn, ${ }^{52,7}$ R. Halliday, ${ }^{49}$ M. R. Hampel, ${ }^{7}$ P. Hansen, ${ }^{60}$ D. Harari, ${ }^{6}$ V. M. Harvey, ${ }^{5}$ A. Haungs, ${ }^{52}$ T. Hebbeker, ${ }^{13}$ D. Heck,${ }^{52}$ G. C. Hill, ${ }^{5}$ C. Hojvat, ${ }^{65}$ J. R. Hörandel,,${ }^{1,37}$ P. Horvath, ${ }^{43}$ M. Hrabovský, ${ }^{43}$ T. Huege, ${ }^{52,31}$ J. Hulsman, ${ }^{7,52}$ A. Insolia, ${ }^{32,33}$ P. G. Isar, ${ }^{73}$ J. A. Johnsen, ${ }^{74}$ J. Jurysek, ${ }^{16}$ A. Kääpä, ${ }^{19}$ K. H. Kampert, ${ }^{19}$ B. Keilhauer, ${ }^{52}$ J. Kemp, ${ }^{13}$ H. O. Klages,${ }^{52}$ M. Kleifges ${ }^{66}$ J. Kleinfeller, ${ }^{40}$ M. Köpke, ${ }^{56}$ G. Kukec Mezek, ${ }^{69}$ B. L. Lago, ${ }^{75}$ D. LaHurd, ${ }^{49}$ R. G. Lang, ${ }^{55}$ N. Langner, ${ }^{13}$ M. A. Leigui de Oliveira, ${ }^{76}$ V. Lenok, ${ }^{52}$ A. Letessier-Selvon, ${ }^{77}$ I. Lhenry-Yvon,${ }^{57}$ D. Lo Presti, ${ }^{32,33}$ L. Lopes, ${ }^{2}$ R. López, ${ }^{78}$ R. Lorek, ${ }^{49}$ Q. Luce, ${ }^{56}$ A. Lucero, ${ }^{7}$ J. P. Lundquist, ${ }^{69}$ A. Machado Payeras, ${ }^{41}$ G. Mancarella, ${ }^{46,39}$ D. Mandat, ${ }^{16}$ B. C. Manning, ${ }^{5}$ J. Manshanden, ${ }^{79}$ P. Mantsch, ${ }^{65}$ S. Marafico, ${ }^{57}$ A. G. Mariazzi, ${ }^{60}$ I. C. Mariş, ${ }^{42}$ G. Marsella, ${ }^{46,39}$ D. Martello, ${ }^{46,39}$ H. Martinez, ${ }^{55}$ O. Martínez Bravo, ${ }^{78}$ M. Mastrodicasa, ${ }^{23,24}$ H. J. Mathes, ${ }^{52}$ J. Matthews, ${ }^{80}$ G. Matthiae, ${ }^{81,82}$ E. Mayotte, ${ }^{19}$ P. O. Mazur, ${ }^{65}$ G. Medina-Tanco, ${ }^{58}$ D. Melo, ${ }^{7}$ A. Menshikov, ${ }^{66}$ K.-D. Merenda ${ }^{74}$ S. Michal ${ }^{43}$ M. I. Micheletti, ${ }^{70}$ L. Miramonti, ${ }^{35,36}$ S. Mollerach, ${ }^{6}$

F. Montanet, ${ }^{20}$ C. Morello, ${ }^{3,4}$ M. Mostafá, ${ }^{83}$ A. L. Müller, ${ }^{7,52}$ M. A. Muller ${ }^{41,25,8}$ K. Mulrey, ${ }^{31}$ R. Mussa, ${ }^{4}$ M. Muzio, ${ }^{64}$ W. M. Namasaka, ${ }^{19}$ L. Nellen, ${ }^{58}$ M. Niculescu-Oglinzanu, ${ }^{17}$ M. Niechciol, ${ }^{29}$ D. Nitz, ${ }^{67, \|}$ D. Nosek,${ }^{84}$ V. Novotny, ${ }^{84}$ L. Nožka, ${ }^{43}$ A. Nucita, ${ }^{46,39}$ L. A. Núñez, ${ }^{85}$ M. Palatka, ${ }^{16}$ J. Pallotta, ${ }^{86}$ P. Papenbreer, ${ }^{19}$ G. Parente, ${ }^{9}$ A. Parra, ${ }^{78}$ M. Pech ${ }^{16}$ F. Pedreira, ${ }^{9}$ J. Pȩkala, ${ }^{27}$ R. Pelayo, ${ }^{87}$ J. Peña-Rodriguez, ${ }^{85}$ J. Perez Armand ${ }^{26}$ M. Perlin, ${ }^{7,52}$ L. Perrone, ${ }^{46,39}$ S. Petrera, ${ }^{47,24}$ T. Pierog, ${ }^{52}$ M. Pimenta, ${ }^{2}$ V. Pirronello, ${ }^{32,33}$ M. Platino, ${ }^{7}$ B. Pont, ${ }^{1}$ M. Pothast,${ }^{37,1}$ P. Privitera, ${ }^{63}$ M. Prouza, ${ }^{16}$ A. Puyleart ${ }^{67}$ S. Querchfeld, ${ }^{19}$ J. Rautenberg, ${ }^{19}$ D. Ravignani, ${ }^{7}$ M. Reininghaus, ${ }^{52,7}$ J. Ridky, ${ }^{16}$ F. Riehn $\odot,{ }^{2}$ M. Risse,${ }^{29}$ P. Ristori, ${ }^{86}$ V. Rizi, ${ }^{23,24}$ W. Rodrigues de Carvalho, ${ }^{26}$ J. Rodriguez Rojo, ${ }^{14}$ M. J. Roncoroni, ${ }^{7}$ M. Roth ${ }^{52}$ E. Roulet, ${ }^{6}$ A. C. Rovero, ${ }^{50}$ P. Ruehl, ${ }^{29}$ S. J. Saffi, ${ }^{5}$ A. Saftoiu, ${ }^{17}$ F. Salamida,${ }^{23,24}$ H. Salazar, ${ }^{78}$ G. Salina, ${ }^{82}$ J. D. Sanabria Gomez,${ }^{85}$ F. Sánchez, ${ }^{7}$ E. M. Santos, ${ }^{26}$ E. Santos, ${ }^{16}$ F. Sarazin, ${ }^{74}$ R. Sarmento, ${ }^{2}$ C. Sarmiento-Cano, ${ }^{7}$ R. Sato, ${ }^{14}$ P. Savina,${ }^{46,39,57}$ C. M. Schäfer, ${ }^{52}$ V. Scherini ${ }^{39}$ H. Schieler, ${ }^{52}$ M. Schimassek ${ }^{56,7}$ M. Schimp, ${ }^{19}$ F. Schlüter, ${ }^{52,7}$ D. Schmidt, ${ }^{56}$ O. Scholten,${ }^{88,31}$ P. Schovánek, ${ }^{16}$ F. G. Schröder, ${ }^{45,52}$ S. Schröder, ${ }^{19}$ J. Schulte, ${ }^{13}$ S. J. Sciutto, ${ }^{60}$ M. Scornavacche, ${ }^{7,52}$ R. C. Shellard ${ }^{89}$ G. Sigl, ${ }^{79}$ G. Silli, ${ }^{7,52}$ O. Sima, ${ }^{17,}$ R. Šmída, ${ }^{63}$ P. Sommers, ${ }^{83}$ J. F. Soriano, ${ }^{11}$ J. Souchard, ${ }^{20}$ R. Squartini, ${ }^{40}$

M. Stadelmaier, ${ }^{52,7}$ D. Stanca, ${ }^{17}$ S. Stanič ${ }^{69}$ J. Stasielak, ${ }^{27}$ P. Stassi, ${ }^{20}$ A. Streich, ${ }^{56,7}$ M. Suárez-Durán, ${ }^{85}$ T. Sudholz, ${ }^{5}$ T. Suomijärvi, ${ }^{22}$ A. D. Supanitsky, ${ }^{7}$ J. Šupík, ${ }^{43}$ Z. Szadkowski, ${ }^{90}$ A. Taboada, ${ }^{56}$ A. Tapia, ${ }^{91}$ C. Timmermans,${ }^{37,1}$ O. Tkachenko, ${ }^{52}$ P. Tobiska, ${ }^{16}$ C. J. Todero Peixoto, ${ }^{38}$ B. Tomé, ${ }^{2}$ G. Torralba Elipe, ${ }^{9}$ A. Travaini, ${ }^{40}$ P. Travnicek, ${ }^{16}$ C. Trimarelli, ${ }^{23,24}$ M. Trini, ${ }^{69}$ M. Tueros, ${ }^{60}$ R. Ulrich,${ }^{52}$ M. Unger,${ }^{52}$ L. Vaclavek,${ }^{43}$ M. Vacula, ${ }^{43}$ J. F. Valdés Galicia, ${ }^{58}$ I. Valiño, ${ }^{47,24}$ L. Valore, ${ }^{18,12}$ E. Varela, ${ }^{78}$ V. Varma K. C., ${ }^{7,52}$ A. Vásquez-Ramírez, ${ }^{85}$ D. Veberič, ${ }^{52}$ C. Ventura, ${ }^{54}$ I. D. Vergara Quispe, ${ }^{60}$ V. Verzi ${ }^{82}$ J. Vicha, ${ }^{16}$ J. Vink,${ }^{92}$ S. Vorobiov, ${ }^{69}$ H. Wahlberg, ${ }^{60}$ A. A. Watson, ${ }^{93}$ M. Weber, ${ }^{66}$ 
A. Weindl,${ }^{52}$ L. Wiencke, ${ }^{74}$ H. Wilczyński, ${ }^{27}$ T. Winchen,${ }^{31}$ M. Wirtz, ${ }^{13}$ D. Wittkowski, ${ }^{19}$ B. Wundheiler, ${ }^{7}$ A. Yushkov, ${ }^{16}$ O. Zapparrata, ${ }^{42}$ E. Zas, ${ }^{9}$ D. Zavrtanik, ${ }^{69,68}$ M. Zavrtanik, ${ }^{68,69}$ L. Zehrer, ${ }^{69}$ and A. Zepeda ${ }^{94}$

(Pierre Auger Collaboration)*

${ }^{1}$ IMAPP, Radboud University Nijmegen, Nijmegen, The Netherlands

${ }^{2}$ Laboratório de Instrumentação e Física Experimental de Partículas-LIP and Instituto Superior Técnico-IST, Universidade de Lisboa-UL, Lisboa, Portugal

${ }^{3}$ Osservatorio Astrofisico di Torino (INAF), Torino, Italy

${ }^{4}$ INFN, Sezione di Torino, Torino, Italy

${ }^{5}$ University of Adelaide, Adelaide, South Australia, Australia

${ }^{6}$ Centro Atómico Bariloche and Instituto Balseiro (CNEA-UNCuyo-CONICET), San Carlos de Bariloche, Argentina

${ }^{7}$ Instituto de Tecnologías en Detección y Astropartículas (CNEA, CONICET, UNSAM), Buenos Aires, Argentina

${ }^{8}$ Universidad Tecnológica Nacional-Facultad Regional Buenos Aires, Buenos Aires, Argentina

${ }^{9}$ Instituto Galego de Física de Altas Enerxías (IGFAE), Universidade de Santiago de Compostela, Santiago de Compostela, Spain

${ }^{10}$ Dipartimento di Fisica, Università Torino, Torino, Italy

${ }^{11}$ Department of Physics and Astronomy, Lehman College, City University of New York, Bronx, New York, USA

${ }^{12}$ INFN, Sezione di Napoli, Napoli, Italy

${ }^{13}$ III. Physikalisches Institut A, RWTH Aachen University, Aachen, Germany

${ }^{14}$ Observatorio Pierre Auger and Comisión Nacional de Energía Atómica, Malargüe, Argentina

${ }^{15}$ University Politehnica of Bucharest, Bucharest, Romania

${ }^{16}$ Institute of Physics of the Czech Academy of Sciences, Prague, Czech Republic

17 "Horia Hulubei" National Institute for Physics and Nuclear Engineering, Bucharest-Magurele, Romania

${ }^{18}$ Dipartimento di Fisica "Ettore Pancini," Università di Napoli "Federico II," Napoli, Italy

${ }^{19}$ Department of Physics, Bergische Universität Wuppertal, Wuppertal, Germany

${ }^{20}$ Université Grenoble Alpes, CNRS, Grenoble Institute of Engineering Université Grenoble Alpes, LPSC-IN2P3, 38000 Grenoble, France

${ }^{21}$ Max-Planck-Institut für Radioastronomie, Bonn, Germany

${ }^{22}$ Université Paris-Saclay, CNRS/IN2P3, IJCLab, Orsay, France

${ }^{23}$ Dipartimento di Scienze Fisiche e Chimiche, Università dell'Aquila, L'Aquila, Italy

${ }^{24}$ INFN Laboratori Nazionali del Gran Sasso, Assergi (L'Aquila), Italy

${ }^{25}$ Instituto de Física, Universidade Federal do Rio de Janeiro, Rio de Janeiro, Brazil

${ }^{26}$ Instituto de Física, Universidade de São Paulo, São Paulo, Brazil

${ }^{27}$ Institute of Nuclear Physics PAN, Krakow, Poland

${ }^{28}$ Colorado State University, Fort Collins, Colorado, USA

${ }^{29}$ Universität Siegen, Fachbereich 7 Physik-Experimentelle Teilchenphysik, Siegen, Germany

${ }^{30}$ Universidad de Granada and C.A.F.P.E., Granada, Spain

${ }^{31}$ Vrije Universiteit Brussels, Brussels, Belgium

${ }^{32}$ Dipartimento di Fisica e Astronomia, Università di Catania, Catania, Italy

${ }^{33}$ INFN, Sezione di Catania, Catania, Italy

${ }^{34}$ Universidad Autónoma de Chiapas, Tuxtla Gutiérrez, Chiapas, México

${ }^{35}$ Dipartimento di Fisica, Università di Milano, Milano, Italy

${ }^{36}$ INFN, Sezione di Milano, Milano, Italy

${ }^{37}$ Nationaal Instituut voor Kernfysica en Hoge Energie Fysica (NIKHEF), Science Park, Amsterdam, The Netherlands

${ }^{38}$ Universidade de São Paulo, Escola de Engenharia de Lorena, Lorena, São Paulo, Brazil

${ }^{39}$ INFN, Sezione di Lecce, Lecce, Italy

${ }^{40}$ Observatorio Pierre Auger, Malargüe, Argentina

${ }^{41}$ Universidade Estadual de Campinas, IFGW, Campinas, São Paulo, Brazil

${ }^{42}$ Université Libre de Bruxelles (ULB), Brussels, Belgium

${ }^{43}$ Palacky University, RCPTM, Olomouc, Czech Republic

${ }^{44}$ Instituto de Tecnologías en Detección y Astropartículas (CNEA, CONICET, UNSAM), and Universidad Tecnológica

Nacional_Facultad Regional Mendoza (CONICET/CNEA), Mendoza, Argentina

${ }^{45}$ Department of Physics and Astronomy, Bartol Research Institute, University of Delaware, Newark, Delaware, USA

${ }^{46}$ Dipartimento di Matematica e Fisica "E. De Giorgi," Università del Salento, Lecce, Italy

${ }^{47}$ Gran Sasso Science Institute, L'Aquila, Italy

${ }^{48}$ Dipartimento di Scienze e Tecnologie Aerospaziali, Politecnico di Milano, Milano, Italy

${ }^{49}$ Case Western Reserve University, Cleveland, Ohio, USA

${ }^{50}$ Instituto de Astronomía y Física del Espacio (IAFE, CONICET-UBA), Buenos Aires, Argentina 


\footnotetext{
${ }^{51}$ Departamento de Física and Departamento de Ciencias de la Atmósfera y los Océanos, FCEyN,

Universidad de Buenos Aires and CONICET, Buenos Aires, Argentina

${ }^{52}$ Karlsruhe Institute of Technology, Institut für Kernphysik, Karlsruhe, Germany

${ }^{53}$ Universidade Federal Fluminense, EEIMVR, Volta Redonda, Rio de Janeiro, Brazil

${ }^{54}$ Universidade Federal do Rio de Janeiro (UFRJ), Observatório do Valongo, Rio de Janeiro, Brazil

${ }^{55}$ Instituto de Física de São Carlos, Universidade de São Paulo, São Carlos, São Paulo, Brazil

${ }^{56}$ Karlsruhe Institute of Technology, Institute for Experimental Particle Physics (ETP), Karlsruhe, Germany

${ }^{57}$ CNRS/IN2P3, IJCLab, Université Paris-Saclay, Orsay, France

${ }^{58}$ Universidad Nacional Autónoma de México, México, Distrito Federal, México

${ }^{59}$ Universidade Federal do Paraná, Setor Palotina, Palotina, Brazil

${ }^{60}$ IFLP, Universidad Nacional de La Plata and CONICET, La Plata, Argentina

${ }^{61}$ Fermi National Accelerator Laboratory, Batavia, Illinois, USA

${ }^{62}$ Stichting Astronomisch Onderzoek in Nederland (ASTRON), Dwingeloo, The Netherlands

${ }^{63}$ University of Chicago, Enrico Fermi Institute, Chicago, Illinois, USA

${ }^{64}$ New York University, New York, New York, USA

${ }^{65}$ Fermi National Accelerator Laboratory, Fermilab, Batavia, Illinois, USA

${ }^{66}$ Karlsruhe Institute of Technology, Institut für Prozessdatenverarbeitung und Elektronik, Karlsruhe, Germany

${ }^{67}$ Michigan Technological University, Houghton, Michigan, USA

${ }^{68}$ Experimental Particle Physics Department, J. Stefan Institute, Ljubljana, Slovenia

${ }^{69}$ Center for Astrophysics and Cosmology (CAC), University of Nova Gorica, Nova Gorica, Slovenia

${ }^{70}$ Instituto de Física de Rosario (IFIR)_CONICET/U.N.R. and Facultad de Ciencias Bioquímicas y Farmacéuticas Universidad Nacional de Rosario, Rosario, Argentina

${ }^{71}$ Faculty of Astrophysics, University of Łódź, Eódź, Poland

${ }^{72}$ Universidade Estadual de Feira de Santana, Feira de Santana, Brazil

${ }^{73}$ Institute of Space Science, Bucharest-Magurele, Romania

${ }^{74}$ Colorado School of Mines, Golden, Colorado, USA

${ }^{75}$ Centro Federal de Educação Tecnológica Celso Suckow da Fonseca, Nova Friburgo, Brazil

${ }^{76}$ Universidade Federal do ABC, Santo André, São Paulo, Brazil

${ }^{77}$ Laboratoire de Physique Nucléaire et de Hautes Energies (LPNHE), Sorbonne Université, Université de Paris, CNRS-IN2P3, Paris, France

${ }^{78}$ Benemérita Universidad Autónoma de Puebla, Puebla, México

${ }^{79}$ Universität Hamburg, II. Institut für Theoretische Physik, Hamburg, Germany

${ }^{80}$ Louisiana State University, Baton Rouge, Louisiana, USA

${ }^{81}$ Dipartimento di Fisica, Università di Roma "Tor Vergata," Roma, Italy

${ }^{82}$ INFN, Sezione di Roma "Tor Vergata," Roma, Italy

${ }^{83}$ Pennsylvania State University, University Park, Pennsylvania, USA

${ }^{84}$ Charles University, Faculty of Mathematics and Physics, Institute of Particle and Nuclear Physics, Prague, Czech Republic

${ }^{85}$ Universidad Industrial de Santander, Bucaramanga, Colombia

${ }^{86}$ Centro de Investigaciones en Láseres y Aplicaciones, CITEDEF and CONICET, Villa Martelli, Argentina

${ }^{87}$ Unidad Profesional Interdisciplinaria en Ingeniería y Tecnologías Avanzadas del Instituto Politécnico Nacional (UPIITA-IPN), México, Distrito Federal, México

${ }^{88}$ KVI-Center for Advanced Radiation Technology, University of Groningen, Groningen, The Netherlands

${ }^{89}$ Centro Brasileiro de Pesquisas Fisicas, Rio de Janeiro, Brazil

${ }^{90}$ Faculty of High-Energy Astrophysics, University of Łódź, Łódź, Poland

${ }^{91}$ Universidad de Medellín, Medellín, Colombia

${ }^{92}$ Faculty of Science, Universiteit van Amsterdam, Amsterdam, The Netherlands

${ }^{93}$ School of Physics and Astronomy, University of Leeds, Leeds, United Kingdom

${ }^{94}$ Centro de Investigación y de Estudios Avanzados del IPN (CINVESTAV), México, Distrito Federal, México
}

(Received 4 October 2020; revised 28 January 2021; accepted 12 February 2021; published 16 April 2021)

We present the first measurement of the fluctuations in the number of muons in extensive air showers produced by ultrahigh energy cosmic rays. We find that the measured fluctuations are in good agreement with predictions from air shower simulations. This observation provides new insights into the origin of the previously reported deficit of muons in air shower simulations and constrains models of hadronic

Published by the American Physical Society under the terms of the Creative Commons Attribution 4.0 International license. Further distribution of this work must maintain attribution to the author(s) and the published article's title, journal citation, and DOI. Funded by $S C O A P^{3}$. 
interactions at ultrahigh energies. Our measurement is compatible with the muon deficit originating from small deviations in the predictions from hadronic interaction models of particle production that accumulate as the showers develop.

DOI: 10.1103/PhysRevLett.126.152002

Introduction.-Ultrahigh energy cosmic rays (UHECRs) are particles coming from outer space, with energies exceeding $10^{18} \mathrm{eV}$. They provide the only experimental opportunity to explore particle physics beyond energies reachable by Earth-based accelerators, which go up to cosmic ray energies of $9 \times 10^{16} \mathrm{eV}$.

The Pierre Auger Observatory [1] detects extensive air showers that are initiated by the UHECRs colliding with the nuclei in the atmosphere. Information about UHECRs is extracted using simulations based on hadronic interaction models which rely on extrapolations of accelerator measurements to unexplored regions of phase space, most notably the forward and highest-energy region. In addition, accelerator experiments at the highest energies either probe the interactions between protons or of protons with heavy nuclei, while most interactions within air showers are between pions and light nuclei.

A further challenge is that the UHECR mass has to be measured despite not being yet completely decoupled from the hadronic uncertainties. The observable with the least dependence on hadronic interactions is the atmospheric depth at which the longitudinal development of the electromagnetic (EM) component of the shower reaches the maximum number of particles, namely, $X_{\max }$ [2].

In hadronic cascades, the energy of each interacting particle is distributed among the secondaries, mostly pions. Neutral pions rapidly decay into two photons, feeding a practically decoupled electromagnetic cascade (other resonances decaying into $\pi^{0}$ 's, electrons, and/or photons also contribute). Charged pions (and other long-lived mesons like kaons) tend to further interact until their individual energies are below a critical value, below which they are more likely to decay. Muons, which are products of hadronic decays, are thus predominantly produced in the final shower stages. In sufficiently inclined showers, the pure EM component is absorbed in the atmosphere and the particles that reach the ground (muons and muon decay products) directly sample the muon content [3,4], reflecting the hadronic component of the shower.

Air showers are mainly detected at the Pierre Auger Observatory by the surface detector (SD), an array of water-Cherenkov detector stations, and the fluorescence detector (FD), consisting of 24 fluorescence telescopes. By selecting the subsample of events reconstructed with both the SD and FD, and with zenith angles exceeding $62^{\circ}$, both the muon content and the energy of the shower are simultaneously measured.

The results obtained indicate that all the simulations underestimate the number of muons in the showers $[5,6]$.
These analyses come with the caveat that they cannot distinguish a muon rescaling from a shift in the absolute energy scale of the FD measurement. However, muon content and energy scale were disentangled in a complementary technique based on showers with zenith angles below $60^{\circ}$. Using the longitudinal profile of the shower in the atmosphere obtained with the FD and the signals at the ground measured with the $\mathrm{SD}$, it was shown that the muonic component still has to be scaled up to match observed data, while no rescaling of the EM component and the FD energy is required [7]. The measurements with the FD also show that both the position of the shower maximum in the atmosphere $\left(X_{\max }\right)$ and the entire shape of the EM shower are well described by the simulations [8,9]. At lower energies, down to $\sim 10^{17.3} \mathrm{eV}$, in a measurement using the subarray of buried scintillators of the Pierre Auger Observatory, a direct count of the muons independent of EM contamination was obtained, which also shows that simulations produce too few muons [10]. There is much evidence that all the simulations underpredict the average number of muons in the showers: a comprehensive study of muon number measurements made with different experiments has shown that the muon deficit in simulations starts around $\sim 10^{16} \mathrm{eV}$ and steadily increases with energy. Depending on model and experiment, the deficit at $\sim 10^{20} \mathrm{eV}$ ranges between tens of percent up to a factor of 2 [11].

The increased statistics obtained at the Pierre Auger Observatory allow us to now take a further step and explore fluctuations in the number of muons between showers, hereinafter referred to as "physical fluctuations." The ratio of the physical fluctuations to the average number of muons (relative fluctuations) has been shown to be mostly dominated by the first interaction, rather than the lower energy interactions deeper in the shower development $[12,13]$. Here, we exploit the sensitivity of fluctuations to the first interaction to explore hadronic interactions well above the energies achievable in accelerator experiments.

Methodology.-Our analysis here is based on the set of inclined air showers $\left(62^{\circ}<\theta<80^{\circ}\right)$ that are reconstructed both with the SD and FD between January 1, 2004 and December 31, 2017. For each event, we obtain independent measurements of the muon content (with the SD) and the calorimetric energy (with the FD). To ensure the showers can be reconstructed with small uncertainties, we select only events with at least four triggered stations in the SD array and we further require that all the stations surrounding the impact point of the shower on the ground are operational at the time of the event. Only events with good atmospheric conditions (few clouds and a low aerosol content) are accepted in order 
to guarantee a good energy reconstruction with the FD. In addition, it is required that the entire shower profile and, in particular, $X_{\max }$ is within the field of view of our telescopes. Since heavy primaries penetrate the atmosphere less than light ones, the acceptance with this selection would be mass dependent. To avoid this bias, we constrain the field of view to the region where all values of $X_{\max }$ are accepted. Further details are given in $[5,14]$. These selection criteria result in a total number of events of 786. In addition, only events with energy larger than $4 \times 10^{18} \mathrm{eV}$, which ensures full trigger efficiency of the SD [3], are used to extract the fluctuations (281 events).

The number of muons is reconstructed by fitting a $2 \mathrm{D}$ model of the lateral profile of the muon density at the ground to the observed signals in the SD array. The free parameters of the fit are the zenith and azimuth angles of the shower, the impact point of the shower on the ground (shower core position), and a normalization factor with respect to a reference muon density profile in simulated proton showers at $10^{19} \mathrm{eV}$ [3]. There exists a residual pure EM component in showers with low zenith angles and stations very close to the shower core position (at $400 \mathrm{~m}$ and $64^{\circ}$ it is $\sim 6 \%$ ), which has been subtracted using a parametrization [4]. The dimensionless normalization factor we obtain from the fit is then transformed to the dimensionless quantity $R_{\mu}$, which is given by the integrated number of muons at the ground divided by a reference given by the average number of muons in simulated proton showers at $10^{19} \mathrm{eV}$ and the given zenith angle. At $10^{19} \mathrm{eV}$ and an inclination of $60^{\circ}, R_{\mu}=1$ corresponds to $2.148 \times 10^{7}$ muons. For more details, see [5]. In the following, we refer to $R_{\mu}$ as the number of muons for short.

The calorimetric energy of the air showers $E_{\text {cal }}$ is reconstructed by integrating the longitudinal shower profiles observed with the FD $[9,15]$. The total energy of the shower is then obtained by adding the average energy carried away by muons and neutrinos, the so-called invisible energy $E=E_{\text {cal }}+E_{\text {inv }}$. At $10^{19} \mathrm{eV}, E_{\text {inv }}$ accounts for $14 \%$ of the total energy in air showers [16-20].

In Fig. 1 the muon number $R_{\mu}$ is shown as a function of the measured energy. Markers on the top of the frame define the bins in energy for which we will extract the fluctuations, with the number of events in each bin shown above. The bins are chosen such that the number of events in each is similar. Based on models of air shower development and given the gradual change of the composition in this energy range (single logarithmic dependence on energy) [8,21-23], the number of muons is related to the primary energy by a single power law

$$
\left\langle R_{\mu}\right\rangle(E)=a\left[E /\left(10^{19} \mathrm{eV}\right)\right]^{b},
$$

which can be fitted following a procedure described in the text below. The best-fit parameters are given at the beginning of the next section. The scattering in the data has three sources: experimental uncertainties in the energy $s_{E}$ and in

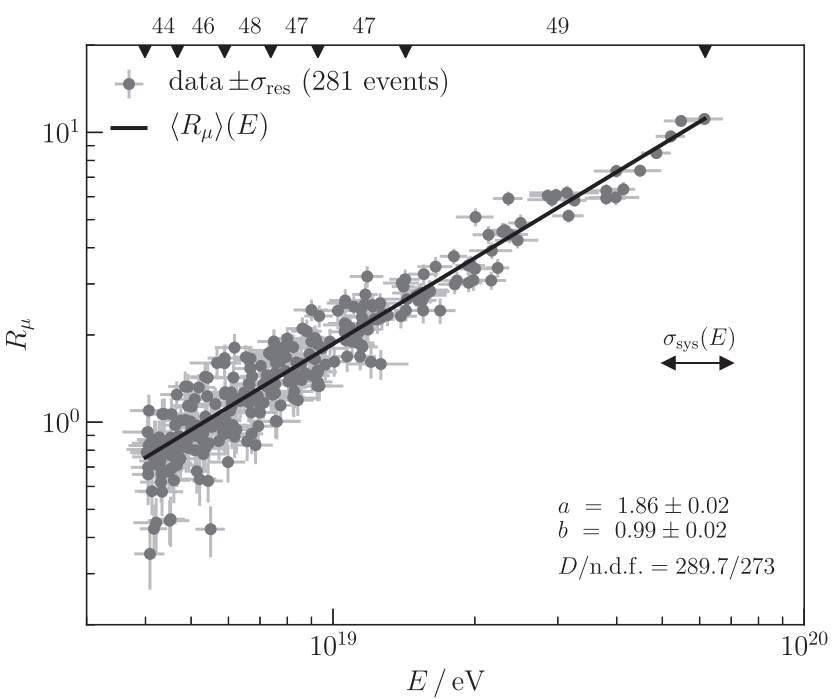

FIG. 1. Number of muons as a function of the measured energy. The black line is the fitted $\left\langle R_{\mu}\right\rangle=a\left[E /\left(10^{19} \mathrm{eV}\right)\right]^{b}$. Markers on the top of the frame define the bins in which the fluctuations are evaluated. The numbers give the events in each bin. The effect of the uncertainty of the absolute energy scale is indicated by $\sigma_{\text {sys }}(E)$. The best-fit values for parameters $a, b$ and the deviance per degree of freedom (n.d.f.) in the fit are shown on the lower right.

the muon number $s_{\mu}$ from event reconstruction (both represented by the error bars), and the physical fluctuations in the muon number denoted as $\sigma$. Given Eq. (1), the variance of the muon number is $\sigma^{2}+b^{2}\left\langle s_{E}\right\rangle^{2}+\left\langle s_{\mu}\right\rangle^{2}$.

In this Letter, we adopt a method based on maximizing the likelihood of a probability distribution function (PDF). The PDF incorporates the various contributions to the fluctuations, treating each energy bin independently while also accounting for the effect of the migration of events between bins [5,24]. The model assumes that measurements of $E$ and $R_{\mu}$ follow Gaussian distributions centered at the true value, with widths given by the detector resolution $s_{E}$ and $s_{\mu}$, which are the uncertainties obtained in each individual event reconstruction [3,25]. Physical fluctuations are also assumed to follow a Gaussian distribution of width $\sigma$. Simulations have shown this is an acceptable approximation given the event number in each bin.

The total PDF is obtained through the convolution of the detector response and the physical fluctuations with the probability distribution of the hybrid events measured at the Pierre Auger Observatory. The log-likelihood function is then given by

$$
\begin{aligned}
\ln \mathcal{L}\left(a, b, \hat{\sigma}_{1}, \ldots, \hat{\sigma}_{6}\right)= & \sum_{i} \ln \left[\sum_{k=0}^{6} \int_{E_{k-1}}^{E_{k}} d E h(E) C(E)\right. \\
& \times \exp \left(-\frac{1}{2} \frac{\left(E_{i}-E\right)^{2}}{s_{E}^{2}}\right) \\
& \left.\times \exp \left(-\frac{1}{2} \frac{\left(R_{\mu, i}-\left\langle R_{\mu}\right\rangle(E)\right)^{2}}{s_{\mu}^{2}+\left(\hat{\sigma}_{k} \cdot\left\langle R_{\mu}\right\rangle(E)\right)^{2}}\right)\right] .
\end{aligned}
$$


The probability of hybrid events $h(E)$ (product of the energy spectrum of cosmic rays and the efficiency of detection) can be obtained from the data, as explained in and $[10,24,26]$. The rhs of Eq. (2) depends on the parameters $a$ and $b$ via Eq. (1). To obtain the energy dependence of the fluctuations, we parametrize $\sigma$ by six independent values such that $\sigma(E)=\hat{\sigma}_{k} \cdot\left\langle R_{\mu}\right\rangle(E)$, where the constants $\hat{\sigma}_{k}$ are the relative fluctuations in the $k$ th energy bin with limits $\left[E_{k-1}, E_{k}\right]$, where $k$ runs from one to six. In Eq. (2), $k=0$ corresponds to the contributions from the interval $\left[0, E_{\mathrm{thr}}\right]$, where the SD is not fully efficient. The fluctuations here are assumed to take the value of the first fitted bin $\hat{\sigma}_{0} \equiv \hat{\sigma}_{1}$.

The sum over the index $i$ in Eq. (2) (the usual sum over the log-likelihoods of events) includes only events above the energy threshold of $4 \times 10^{18} \mathrm{eV}$. The function $C(E)$ is the normalization factor from the double Gaussian. The result of the fit for the parameters $a$ and $b$ are shown in Fig. 1. The fluctuations are shown in Fig. 2. The distribution of the number of muons and the PDF in the individual energy bins can be found in the Supplemental Material [17].

The dominant systematic uncertainties of $\sigma$ come from the uncertainties in the resolutions $s_{E}$ and $s_{\mu}$. For $s_{\mu}$ we estimate the uncertainty using simulations and data. In simulations, the uncertainty was estimated by the spread in a sample of simulated showers, where each shower is reconstructed multiple times, each time changing only the impact point at the ground. For data, we reconstruct the same event multiple times, leaving out the signals from one of the detector stations. The average relative resolution

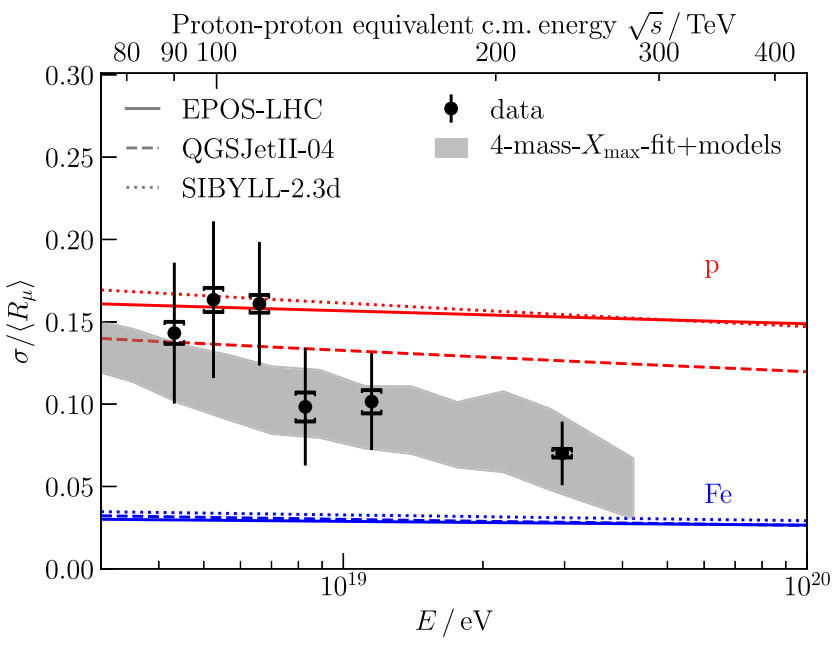

FIG. 2. Measured relative fluctuations in the number of muons as a function of the energy and the predictions from three interaction models for proton (red) and iron (blue) showers. The gray band represents the expectations from the measured mass composition interpreted with the interaction models. The statistical uncertainty in the measurement is represented by the error bars. The total systematic uncertainty is indicated by the square brackets. $\left\langle s_{\mu} / R_{\mu}\right\rangle$ and its systematic uncertainty is thus $(10 \pm 3) \%$ at $10^{19} \mathrm{eV}$.

We verified the values of $s_{E}$ by studying the difference in the energy reconstruction of events measured independently by two or more FD stations. The width of the distribution of these energy differences is found to be compatible with $s_{E}$. We therefore take the statistical $1-\sigma$ uncertainties of this cross check as a conservative upper limit of the systematic uncertainty of $s_{E}$ [27]. The average relative energy resolution $\left\langle s_{E} / E\right\rangle$ is about $(8.4 \pm 2.9) \%$ at $10^{19} \mathrm{eV}$. We have further confirmed that there are no significant contributions to the fluctuations from differences between the individual FD stations, neither related to the longtime performance evolution of the SD and FD detectors.

Any residual electromagnetic component in the signal would affect the lower zenith angles more. We therefore split the event sample at the median zenith angle $\left(66^{\circ}\right)$ and compare the resulting fluctuations. We find no significant difference between the more and the less inclined sample.

In another test, we do find a small modulation of $\left\langle R_{\mu}\right\rangle$ with the azimuth angle $(<1 \%)$, which we correct for. This modulation is related to the approximations used in the reconstruction, which deal with the azimuthal asymmetry of the muon densities at the ground due to the Earth's magnetic field [3]. Finally, we have run an end-to-end validation of the whole analysis method described in this Letter on samples of simulated proton, helium, oxygen, and iron showers.

Because of the almost linear relation between $R_{\mu}$ and $E$, the systematic uncertainty on $\sigma$ due to the uncertainty of the absolute energy scale of $14 \%$ [25] practically cancels out in the relative fluctuations. The systematic uncertainty in the absolute scale of $R_{\mu}$ of $11 \%$ [5] drops out for the same reason. The systematic effects for the bin around $10^{19} \mathrm{eV}$ are summarized in Table I. Over all energies, the systematic uncertainties are below $8 \%$.

Results and discussion.-The best-fit value for the average relative number of muons at $10^{19} \mathrm{eV}$ (parameter $a$ ) is $\left\langle R_{\mu}\right\rangle\left(10^{19} \mathrm{eV}\right)=1.86 \pm 0.02$ (stat) ${ }_{-0.31}^{+0.36}$ (syst). For the slope (parameter $b$ ) we find $d\left\langle\ln R_{\mu}\right\rangle / d \ln E=0.99 \pm$ 0.02 (stat) ${ }_{-0.03}^{+0.03}$ (syst). These values are consistent with the values previously reported $[5,17]$.

TABLE I. Contributions to the systematic uncertainty in the relative fluctuations around $10^{19} \mathrm{eV}\left(10^{18.97}-10^{19.15} \mathrm{eV}\right)$. The central value is $\sigma /\left\langle R_{\mu}\right\rangle=0.102 \pm 0.029$ (stat) \pm 0.007 (syst).

\begin{tabular}{lcc}
\hline \hline Source of uncertainty & & Uncertainty (\%) \\
\hline$E$ absolute scale & $\langle E\rangle$ & $<0.1$ \\
$E$ resolution & $S_{E}$ & 4.6 \\
$R_{\mu}$ absolute scale & $\left\langle R_{\mu}\right\rangle$ & 0.5 \\
$R_{\mu}$ resolution & $S_{\mu}$ & 5.2 \\
$R_{\mu}$ azimuthal modulation & $\left\langle R_{\mu}\right\rangle(\phi)$ & 0.5 \\
Total systematics & & 7.0 \\
\hline \hline
\end{tabular}


The measured relative fluctuations as a function of the energy are shown in Fig. 2. We note that the measurement falls within the range that is expected from current hadronic interaction models for pure proton and pure iron primaries [28-36]. To estimate the effect of a mixed composition, we take the fractions of the four mass components (proton, helium, nitrogen, and iron) derived from the $X_{\max }$ measurements $[8,37,38]$ and, using the simulations of the pure primaries, calculate the corresponding fluctuations in the number of muons. The gray band in Fig. 2 encompasses the predicted $\sigma /\left\langle R_{\mu}\right\rangle$ of the three interaction models QGSJET II-04, EPOS-LHC, and Sibyll 2.3d given the inferred composition mix for each [17].

In Fig. 3, the effects of different composition scenarios on both the fluctuations and the average number of muons can be shown by drawing, at a fixed primary energy of $10^{19} \mathrm{eV}$, the relative fluctuations $\sigma /\left\langle R_{\mu}\right\rangle$ against the average number of muons $\left\langle R_{\mu}\right\rangle$. Given any one of the interaction models, any particular mixture of the four components $p, \mathrm{He}, \mathrm{N}$, and Fe falls somewhere within one of the areas enclosed by the corresponding colored lines. The points of pure composition in this contour are labeled accordingly. For each model, the expected values for $\sigma /\left\langle R_{\mu}\right\rangle$ and $\left\langle R_{\mu}\right\rangle$ given the composition mixture obtained from the $X_{\max }$ measurements [8] is indicated within each contour by the correspondingly colored star marker. The shaded areas surrounding the star markers

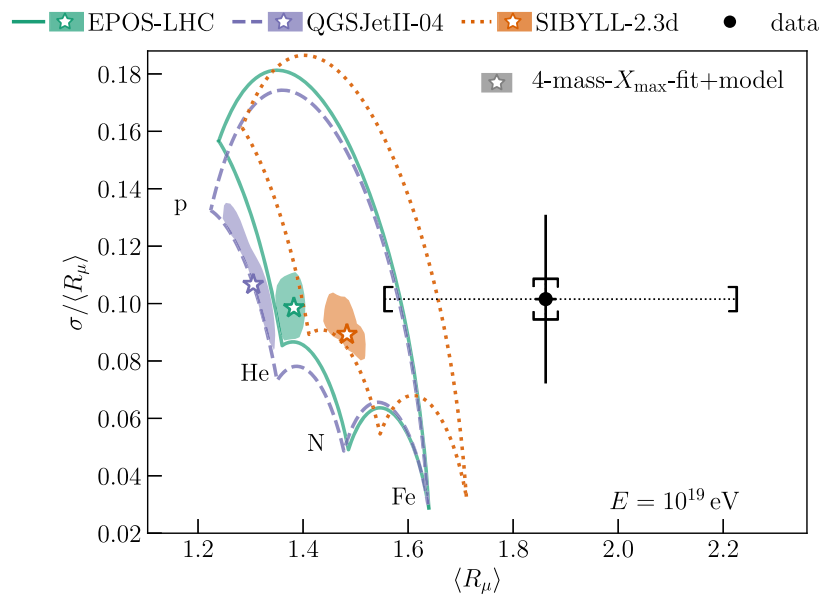

FIG. 3. Data (black, with error bars) compared to models for the fluctuations and the average number of muons for showers with a primary energy of $10^{19} \mathrm{eV}$. Fluctuations are evaluated in the energy range from $10^{18.97}$ to $10^{19.15} \mathrm{eV}$. The statistical uncertainty is represented by the error bars. The total systematic uncertainty is indicated by the square brackets. The expectation from the interaction models for any mixture of the four components $p, \mathrm{He}, \mathrm{N}, \mathrm{Fe}$ is illustrated by the colored contours. The values preferred by the mixture derived from the $X_{\max }$ measurements are indicated by the star symbols. The shaded areas show the regions allowed by the statistical and systematic uncertainties of the $X_{\max }$ measurement [39]. indicate the statistical and systematic uncertainties inherited from the $X_{\max }$ measurements [39]. Finally, our measurement with statistical and systematic uncertainty is shown by the black marker.

Within the uncertainty, none of the predictions from the interaction models and the $X_{\max }$ composition (star markers) are consistent with our measurement. The predictions from the interaction models QGSJET II-04, EPOS-LHC, and Sibyll $2.3 \mathrm{~d}$ can be reconciled with our measurement by an increase in the average number of muons of $43 \%, 35 \%$, and $26 \%$, respectively. For the fluctuations, no rescaling is necessary for any model.

Taken together, the average value and fluctuations of the muon flux constrain the way hadronic interaction models should be changed to agree with air shower data. To see this, we briefly discuss the origin of the fluctuations.

The average number of muons in a proton shower of energy $E$ has been shown in simulations to scale as $\left\langle N_{\mu}^{*}\right\rangle=C E^{\beta}$, where $\beta \simeq 0.9[12,13,22,23]$. If we assume all the secondaries from the first interaction produce muons following the same relation as given for protons above, we obtain the number of muons in the shower as

$$
N_{\mu}=\sum_{j=1}^{m} C E_{j}^{\beta}=\left\langle N_{\mu}^{*}\right\rangle \sum_{j=1}^{m} x_{j}^{\beta}=\left\langle N_{\mu}^{*}\right\rangle \alpha_{1}
$$

where index $j$ runs over $m$ secondary particles which reinteract hadronically and $x_{j}=E_{j} / E$ is the fraction of energy fed to the hadronic shower by each [41]. In this expression, the fluctuations in $N_{\mu}$ are induced by $\alpha_{1}$ in the first generation, which fluctuates because the multiplicity $m$ and the energies $x_{j}$ of the secondaries fluctuate [13].

We can continue this reasoning for the subsequent generations to obtain

$$
\frac{N_{\mu}}{\left\langle N_{\mu}^{*}\right\rangle}=\alpha_{1} \alpha_{2} \cdots \alpha_{i} \cdots \alpha_{n}
$$

here the subindex $i$ runs over $n$ generations, until the cascade stops. We note that, for the calculation of $\alpha_{2}$, in the second generation, there are $m$ particles contributing. Assuming the distributions of the $\alpha$ 's for each one are similar, when adding up the muons produced by each, the fluctuations produced by one are statistically likely to be compensated by another. In other words, the $\alpha_{2}$ distribution is narrower by a factor $\sim 1 / \sqrt{m}$. The deeper the generation, the sharper the corresponding $\alpha_{i}$ is expected to be. As a result, the dominant part of the fluctuations comes from the first interaction. This has also been observed with simulations. The model can be generalized for primary nuclei with mass $A$ using the superposition model and fixing the number of participants to $A$ protons, which reduces the different contributions to the fluctuations by a factor $\sim 1 / \sqrt{A}$. 
There are two options to increase the average number of muons in air showers. One is to increase $\alpha$ in a specific generation, notably the first where the energy is the highest and exotic phenomena could conceivably play a role, i.e., $\alpha_{1} \rightarrow \alpha_{1}+\delta \alpha_{1}$. Note that, if only the first generation is modified (implying some sort of threshold effect for new physics), the increase in $N_{\mu}$ is linear with the modification. There are several examples in the literature where this approach has been used assuming different mechanisms [43-47]. For the fluctuations, the change depends on the model. Alternatively, the number of muons can be increased by introducing small deviations in the hadronic energy fraction $\delta \alpha$ in all generations. Accumulated along a number $n$ of generations, these small deviations build up as $N_{\mu} \propto(\alpha+\delta \alpha)^{n}$. For instance, a 5\% deviation per generation converts into $\sim 30 \%$ deviation after six generations [48]. On the other hand, a change of 5\% in the fluctuations of $\alpha$ is not amplified in the muon fluctuations because of the suppression in later generations. This approach characterizes the increase in the number of muons in the current hadronic interaction models with regard to previous models [32,50-54]. It is also compatible with the increase of the discrepancy in the average number of muons across a wide range of energies reported in [11].

The present analysis finding that fluctuations are consistent with model predictions means that the increase in muon number may be a small effect accumulating over many generations or a very particular modification of the first interaction that changes $N_{\mu}$ without changing the fluctuations [17].

Summary.-We have presented for the first time a measurement of the fluctuations in the number of muons in inclined air showers, as a function of the UHECR primary energy. Within the current uncertainties, the relative fluctuations show no discrepancy with respect to the expectation from current high-energy hadronic interaction models and the composition taken from $X_{\max }$ measurements. This agreement between models and data for the fluctuations, combined with the significant deficit in the predicted total number of muons, points to the origin of the models' muon deficit being a small deficit at every stage of the shower that accumulates along the shower development, rather than a discrepancy in the first interaction. Adjustments to models to address the current muon deficit must therefore not alter the predicted relative fluctuations.

The Pierre Auger Observatory is currently undergoing an upgrade that includes the deployment of scintillators on top of the SD stations [55] to help disentangle the muonic and electromagnetic content of the showers, as well as an array of radio antennas [56]. It has been shown that radio arrays can provide an estimate of the calorimetric energy [57], and therefore, it will soon be possible to perform an analysis similar to the one presented here with much larger statistics using hybrid events measured by the high-duty-cycle radio and surface detector arrays [56].
The successful installation, commissioning, and operation of the Pierre Auger Observatory would not have been possible without the strong commitment and effort from the technical and administrative staff in Malargüe. We are very grateful to the following agencies and organizations for financial support: Argentina-Comisión Nacional de Energía Atómica, Agencia Nacional de Promoción Científica y Tecnológica (ANPCyT), Consejo Nacional de Investigaciones Científicas y Técnicas (CONICET), Gobierno de la Provincia de Mendoza, Municipalidad de Malarguie, NDM Holdings and Valle Las Leñas; in gratitude for their continuing cooperation over land access; Australia-the Australian Research Council; BrazilConselho Nacional de Desenvolvimento Científico e Tecnológico (CNPq), Financiadora de Estudos e Projetos (FINEP), Fundação de Amparo à Pesquisa do Estado de Rio de Janeiro (FAPERJ), São Paulo Research Foundation (FAPESP) Grants No. 2019/10151-2, No. 2010/07359-6, and No. 1999/05404-3, Ministério da Ciência, Tecnologia, Inovações e Comunicações (MCTIC); Ministry of Education, Youth and Sports of the Czech RepublicGrants No. MSMT CR LTT18004, No. LM2015038, No. LM2018102, No. CZ.02.1.01/0.0/0.0/16_013/ 0001402, No. CZ.02.1.01/0.0/0.0/18_046/0016010, and No. CZ.02.1.01/0.0/0.0/17_049/0008422; France-Centre de Calcul IN2P3/CNRS, Centre National de la Recherche Scientifique (CNRS), Conseil Régional Ile-de-France, Département Physique Nucléaire et Corpusculaire (PNC-IN2P3/CNRS), Département Sciences de l'Univers (SDU-INSU/CNRS), Institut Lagrange de Paris (ILP) Grant No. LABEX ANR-10-LABX-63 within the Investissements d'Avenir Programme Grant No. ANR11-IDEX-0004-02; Germany-Bundesministerium für Bildung und Forschung (BMBF), Deutsche Forschungsgemeinschaft (DFG), Finanzministerium Baden-Württemberg, Helmholtz Alliance for Astroparticle Physics (HAP), Helmholtz-Gemeinschaft Deutscher Forschungszentren (HGF), Ministerium für Innovation, Wissenschaft und Forschung des Landes Nordrhein-Westfalen, Ministerium für Wissenschaft, Forschung und Kunst des Landes Baden-Württemberg; Italy-Istituto Nazionale di Fisica Nucleare (INFN), Istituto Nazionale di Astrofisica (INAF), Ministero dell'Istruzione, dell'Universitá e della Ricerca (MIUR), CETEMPS Center of Excellence, Ministero degli Affari Esteri (MAE); México-Consejo Nacional de Ciencia y Tecnología (CONACYT) Grant No. 167733, Universidad Nacional Autónoma de México (UNAM), PAPIIT DGAPA-UNAM; The Netherlands-Ministry of Education, Culture and Science, Netherlands Organisation for Scientific Research (NWO), Dutch national e-infrastructure with the support of SURF Cooperative; Poland-Ministry of Science and Higher Education, Grant No. DIR/WK/2018/11, National Science Centre, Grants No. 2013/08/M/ST9/00322, 
No. 2016/23/B/ST9/01635, and No. HARMONIA 5-2013/ 10/M/ST9/00062, UMO-2016/22/M/ST9/00198; Portugal -Portuguese national funds and FEDER funds within Programa Operacional Factores de Competitividade through Fundação para a Ciência e a Tecnologia (COMPETE); Romania-Romanian Ministry of Education and Research, the Program Nucleu within MCI (PN19150201/16N/2019 and PN19060102), and project PN-III-P1-1.2-PCCDI-2017-0839/19PCCDI/2018 within PNCDI III; Slovenia-Slovenian Research Agency, Grants No. P1-0031, No. P1-0385, No. I00033, No. N1-0111; Spain-Ministerio de Economía, Industria y Competitividad (FPA2017-85114-P and FPA2017-85197-P), Xunta de Galicia (ED431C 2017/ 07), Junta de Andalucía (SOMM17/6104/UGR), Feder Funds, RENATA Red Nacional Temática de Astropartículas (FPA2015-68783-REDT), and María de Maeztu Unit of Excellence (MDM-2016-0692); U.S.Department of Energy, Awards No. DE-AC0207CH11359, No. DE-FR02-04ER41300, No. DE-FG0299ER41107, and No. DE-SC0011689, National Science Foundation, Grant No. 0450696, The Grainger Foundation, Marie Curie-IRSES/EPLANET, European Particle Physics Latin American Network, and UNESCO.

*auger_spokespersons@fnal.gov; http://www.auger.org ${ }^{\dagger}$ Also at Radboud Universtiy Nijmegen, Nijmegen, The Netherlands.

${ }^{*}$ Present address: Hakubi Center for Advanced Research and Graduate School of Science, Kyoto University, Kyoto, Japan.

${ }^{\S}$ Also at Universidade Federal de Alfenas, Poços de Caldas, Brazil.

"Also at Karlsruhe Institute of Technology, Karlsruhe, Germany.

"Also at University of Bucharest, Physics Department, Bucharest, Romania.

[1] A. Aab et al. (Pierre Auger Collaboration), The Pierre Auger Cosmic Ray Observatory, Nucl. Instrum. Methods Phys. Res., Sect. A 798, 172 (2015).

[2] J. Linsley, Structure of large showers at depth $834 \mathrm{~g} / \mathrm{cm}^{2}$, applications, in Proceedings of the 15th Int. Cosmic Ray Conf., Plovdiv, Bulgaria (Bulgarian Academy of Sciences, Bulgaria, 1977), Vol. 12, p. 89.

[3] A. Aab et al. (Pierre Auger Collaboration), Reconstruction of inclined air showers detected with the Pierre Auger Observatory, J. Cosmol. Astropart. Phys. 08 (2014) 019.

[4] I. Valiño, J. Alvarez-Muñiz, M. Roth, R. A. Vazquez, and E. Zas, Characterisation of the electromagnetic component in ultra-high energy inclined air showers, Astropart. Phys. 32, 304 (2010).

[5] A. Aab et al. (Pierre Auger Collaboration), Muons in air showers at the Pierre Auger Observatory: Mean number in highly inclined events, Phys. Rev. D 91, 032003 (2015); Erratum, Phys. Rev. D91, 059901 (2015).

[6] S. J. Sciutto, Air showers, hadronic models, and muon production, EPJ Web Conf. 210, 02007 (2019).
[7] A. Aab et al. (Pierre Auger Collaboration), Testing Hadronic Interactions at Ultrahigh Energies with Air Showers Measured by the Pierre Auger Observatory, Phys. Rev. Lett. 117, 192001 (2016).

[8] A. Aab et al. (Pierre Auger Collaboration), Depth of maximum of air-shower profiles at the Pierre Auger Observatory. I. Measurements at energies above $10^{17.8} \mathrm{eV}$, Phys. Rev. D 90, 122005 (2014).

[9] A. Aab et al. (Pierre Auger Collaboration), Measurement of the average shape of longitudinal profiles of cosmic-ray air showers at the Pierre Auger Observatory, J. Cosmol. Astropart. Phys. 03 (2019) 018.

[10] A. Aab et al. (Pierre Auger Collaboration), Direct measurement of the muonic content of extensive air showers between $\mathbf{2} \times \mathbf{1 0}^{\mathbf{1 7}}$ and $\mathbf{2} \times \mathbf{1 0}^{\mathbf{1 8}} \mathrm{eV}$ at the Pierre Auger Observatory, Eur. Phys. J. C 80, 751 (2020).

[11] L. Cazon et al. (EAS-MSU1, IceCube, KASCADE-Grande, NEVOD-DECOR, Pierre Auger, SUGAR, Telescope Array, and Yakutsk EASArray Collaborations), Working group report on the combined analysis of muon density measurements from eight air shower experiments, Proc. Sci., ICRC2019 (2019) 214.

[12] S. Fukui, H. Hasegawa, T. Matano, I. Miura, M. Oda, K. Suga, G. Tanahashi, and Y. Tanaka, A study on the structure of the extensive air shower, Prog. Theor. Phys. Suppl. 16, 1 (1960).

[13] L. Cazon, R. Conceição, and F. Riehn, Probing the energy spectrum of hadrons in proton air interactions at ultrahigh energies through the fluctuations of the muon content of extensive air showers, Phys. Lett. B 784, 68 (2018).

[14] P. Abreu, M. Aglietta, E. J. Ahn, D. Allard, I. Allekotte, J. Allen, J. Alvarez Castillo, J. Alvarez-Muñiz, M. Ambrosio, A. Aminaei et al., The exposure of the hybrid detector of the Pierre Auger Observatory, Astropart. Phys. 34, 368 (2011).

[15] J. Abraham et al. (Pierre Auger Collaboration), The fluorescence detector of the Pierre Auger Observatory, Nucl. Instrum. Methods Phys. Res., Sect. A 620, 227 (2010).

[16] A. Aab et al. (Pierre Auger Collaboration), Data-driven estimation of the invisible energy of cosmic ray showers with the Pierre Auger Observatory, Phys. Rev. D 100, 082003 (2019).

[17] See Supplemental Material at http://link.aps.org/ supplemental/10.1103/PhysRevLett.126.152002 for additional information on the energy measurement, the comparison of the full model distribution for $R_{\mu}$ in the individual energy bins, updated versions of select figures from [5], the predictions by the individual models, and additional information on the average number of muons and the fluctuations.

[18] H. M. J. Barbosa, F. Catalani, J. A. Chinellato, and C. Dobrigkeit, Determination of the calorimetric energy in extensive air showers, Astropart. Phys. 22, 159 (2004).

[19] M. Risse and D. Heck, Energy release in air showers, Astropart. Phys. 20, 661 (2004).

[20] T. Pierog, M. Alekseeva, T. Bergmann, V. Chernatkin, R. Engel, D. Heck, N. N. Kalmykov, S. Ostapchenko, M. Unger, and K. Werner, Dependence of the longitudinal shower profile on the characteristics of hadronic multiparticle production, Int. Cosmic Ray Conf. 7, 103 (2005), 
https://ui.adsabs.harvard.edu/link_gateway/2005ICRC....7. .103P/ADS_PDF.

[21] K.-H. Kampert and M. Unger, Measurements of the cosmic ray composition with air shower experiments, Astropart. Phys. 35, 660 (2012).

[22] J. Matthews, A Heitler model of extensive air showers, Astropart. Phys. 22, 387 (2005).

[23] R. Engel, D. Heck, and T. Pierog, Extensive air showers and hadronic interactions at high energy, Annu. Rev. Nucl. Part. Sci. 61, 467 (2011).

[24] H. P. Dembinski, B. Kégl, I. C. Mariş, M. Roth, and D. Veberič, A likelihood method to cross-calibrate air-shower detectors, Astropart. Phys. 73, 44 (2016).

[25] B. Dawson et al. (Pierre Auger Collaboration), The energy scale of the Pierre Auger Observatory, Proc. Sci., ICRC2019 (2019) 231, https://pos.sissa.it/358/231/pdf.

[26] A. Aab et al. (Pierre Auger Collaboration), Measurement of the cosmic-ray energy spectrum above $2.5 \times 10^{18} \mathrm{eV}$ using the Pierre Auger Observatory, Phys. Rev. D 102, 062005 (2020).

[27] The resolution systematics estimated in [25] are smaller by about a factor 3 , but were derived for different quality cuts than the ones applied here.

[28] T. Pierog and K. Werner, EPOS model and ultra high energy cosmic rays, Nucl. Phys. B, Proc. Suppl. 196, 102 (2009).

[29] T. Pierog, Iu. Karpenko, J. M. Katzy, E. Yatsenko, and K. Werner, EPOS LHC: Test of collective hadronization with data measured at the CERN Large Hadron Collider, Phys. Rev. C 92, 034906 (2015).

[30] S. Ostapchenko, Monte Carlo treatment of hadronic interactions in enhanced Pomeron scheme: I. QGSJET-II model, Phys. Rev. D 83, 014018 (2011).

[31] E.-J. Ahn, R. Engel, T. K. Gaisser, P. Lipari, and T. Stanev, Cosmic ray interaction event generator Sibyll 2.1, Phys. Rev. D 80, 094003 (2009).

[32] F. Riehn, R. Engel, A. Fedynitch, T. K. Gaisser, and T. Stanev, Hadronic interaction model Sibyll 2.3d and extensive air showers, Phys. Rev. D 102, 063002 (2020).

[33] T. Bergmann, R. Engel, D. Heck, N. Kalmykov, S. Ostapchenko, T. Pierog, T. Thouw, and K. Werner, One-dimensional hybrid approach to extensive air shower simulation, Astropart. Phys. 26, 420 (2007).

[34] T. Pierog et al., First results of fast one-dimensional hybrid simulation of EAS using CONEX, Nucl. Phys. B, Proc. Suppl. 151, 159 (2006).

[35] A. Ferrari et al., FLUKA: A multi-particle transport code, Reports No. CERN-2005-010, No. SLAC-R-773, No. INFN-TC-05-11, 2005.

[36] T. T. Böhlen, F. Cerutti, M. P. W. Chin, A. Fassò, A. Ferrari, P. G. Ortega, A. Mairani, P. R. Sala, G. Smirnov, and V. Vlachoudis, The FLUKA code: Developments and challenges for high energy and medical applications, Nucl. Data Sheets 120, 211 (2014).

[37] J. Bellido (Pierre Auger Collaboration), Depth of maximum of air-shower profiles at the Pierre Auger Observatory: Measurements above $10^{17.2} \mathrm{eV}$ and composition implications, Proc. Sci., ICRC2017 (2018) 506, https://pos.sissa.it/ 301/506/pdf.
[38] The mass fractions that are used for Sibyll here were derived for Sibyll 2.3. Given the small difference of $5 \mathrm{~g} / \mathrm{cm}^{2}$ in $\left\langle X_{\max }\right\rangle$ and $\sigma\left(X_{\max }\right)$ between Sibyll 2.3 and Sibyll 2.3d, the mass fractions for Sibyll $2.3 \mathrm{~d}$ are expected to be very similar.

[39] While the $68 \%$ contours of EPOS and Sibyll cover a range of different mixtures, the QGSJET contour is elongated along the p-He line. The latter is an artifact originating from the poor description of the measured $X_{\max }$ distribution with the QGSJET model [40].

[40] A. Aab et al. (Pierre Auger Collaboration), Depth of maximum of air-shower profiles at the Pierre Auger Observatory. II. Composition implications, Phys. Rev. D 90, 122006 (2014).

[41] The energy fed to the electromagnetic shower in the first interaction is $E-\sum_{j=1}^{m} E_{j}$, and it rapidly decreases for next generations [42].

[42] L. Cazon, Probing high-energy hadronic interactions with extensive air showers, Proc. Sci., ICRC2019 (2019) 005 [arXiv:1909.02962].

[43] R. Aloisio, D. Boncioli, A. di Matteo, P. L. Ghia, A. F. Grillo, S. Petrera, and F. Salamida, Are cosmic rays still a valuable probe of Lorentz invariance violations in the Auger era? Frascati Phys. Ser. 58, 274 (2014), http://www.lnf.infn .it/sis/frascatiseries/Volume58/Volume58.pdf.

[44] J. Alvarez-Muñiz, L. Cazon, R. Conceição, J. Dias de Deus, C. Pajares, and M. Pimenta, Muon production and string percolation effects in cosmic rays at the highest energies, arXiv:1209.6474.

[45] L. A. Anchordoqui, H. Goldberg, and T. J. Weiler, Strange fireball as an explanation of the muon excess in Auger data, Phys. Rev. D 95, 063005 (2017).

[46] G. R. Farrar, Particle physics at ultrahigh energies, in Proceedings, 18th International Symposium on Very High Energy Cosmic Ray Interactions (ISVHECRI 2014): Geneva, Switzerland, August 18-22, 2014 (2019) [arXiv:1902.11271].

[47] G. R. Farrar and J. D. Allen, A new physical phenomenon in ultra-high energy collisions, EPJ Web Conf. 53, 07007 (2013).

[48] The number of generations is difficult to define, as it depends on the details of the measurement and the showers, like the zenith angle, the muon energy threshold, and distance from shower axis. Six is a reasonable minimum of generations before the muons reach the Auger detectors $[22,49]$.

[49] J. Alvarez-Muñiz, R. Engel, T. K. Gaisser, J. A. Ortiz, and T. Stanev, Hybrid simulations of extensive air showers, Phys. Rev. D 66, 033011 (2002).

[50] P. K. F. Grieder, The effect of $n \bar{n}$-production on particle spectra in vertically incident and inclined showers derived from simulations, in Proceedings of 13th International Cosmic Ray Conference, Denver (1973), Vol. 4.

[51] T. Pierog and K. Werner, Muon Production in Extended Air Shower Simulations, Phys. Rev. Lett. 101, 171101 (2008).

[52] H.-J. Drescher, Remnant break-up and muon production in cosmic ray air showers, Phys. Rev. D 77, 056003 (2008). 
[53] S. Ostapchenko, QGSJET-II: physics, recent improvements, and results for air showers, EPJ Web Conf. 52, 02001 (2013).

[54] M. Rybczyński and Z. Włodarczyk, Puzzle of muons in extensive air showers, Int. J. Mod. Phys. D 28, 1950097 (2019).

[55] A. Aab et al. (Pierre Auger Collaboration), The Pierre Auger Observatory upgrade-Preliminary design report, Report No. FERMILAB-DESIGN-2016-05, arXiv:1604.03637.
[56] B. Pont et al. (Pierre Auger Collaboration), A large radio detector at the Pierre Auger Observatory-Measuring the properties of cosmic rays up to the highest energies, Proc. Sci., ICRC2019 (2019) 395.

[57] A. Aab et al. (Pierre Auger Collaboration), Measurement of the Radiation Energy in the Radio Signal of Extensive Air Showers as a Universal Estimator of Cosmic-Ray Energy, Phys. Rev. Lett. 116, 241101 (2016). 